\title{
Ultimate strength of adjustable telescopic steel props according to standard EN 1065.
}

\author{
A. Salvadori, E. Marchina \\ CeSiA - Centro di studio e ricerca di sismologia applicata e dinamica strutturale, \\ DICATA - Dipartimento di Ingegneria Civile, Architettura, Territorio e Ambiente \\ Università di Brescia, via Branze 43, 25123 Brescia, Italy \\ E-mail: alberto.salvadori@ing.unibs.it; egidio.marchina@ing.unibs.it
}

\begin{abstract}
The European standard EN 1065 - to which most current European production appears not to have been upgraded - addresses the design and the manufacturing of adjustable telescopic steel props, an interest bearing economic sector. The present work aims at showing an efficient strategy for the assessment of the ultimate strength of steel props according to EN 1065 in order to identify the ideal cost-performance ratio in the real life production.
\end{abstract}

\section{Introduction}

The European standard EN-1065 specifies materials, design requirements, and protection alternatives against the corrosion together with validation methods using both calculations and tests for adjustable telescopic steel props (from now on called simply props) with threading covered or uncovered. It addresses their production, design and validation through calculations and tests, ruling an interest bearing economic sector. Noteworthy, the current European production generally does not comply the standard in terms of dimensional requirements: therefore the optimal cost-performance product design seems to be mandatory. Since the raw material cost is of fundamental incidence, the minimal weight has to be pursued in the production. This target can be reached through an effective evaluation of the carrying capacity: this is the aim of the present note. It concerns the structural design of props, in order to minimize the safety factor $s>1$ with respect to the collapse mechanisms [1] defined in the standard EN 1065.

Paragraph 2 summarizes the standard requirements for the design of the tubes; the classification based on the strength; the configurations of the components of each class, which cause strong dimensional constraints to the production; the criteria for the evaluation of the ultimate loading capacity and the collapse mechanisms considered by the standard; the static models; the description of the constructive flaws and their modeling; the tolerances; the materials.

The next paragraph deals with an algorithm for the evaluation of the carrying capacity. It stems from the interpretation of the non linear constitutive law that models the base of a prop. The algorithm allows: i) the exact description of the "three steps" structural evolution; ii) the Eulerian critical loads; iii) the evaluation of the loads capable to activate each collapse mechanisms considered by the standard: the lowest of them is defined as the ultimate strength of a prop. This analytical approach seems to be characteristic of the present note, whereas different methodologies have been authoritatively proposed in the literature [2].

The algorithm is finally applied in paragraph 4 to the evaluation of the carrying capacity of a prop, with features taken from the real life production. A critical analysis of standard EN-1065, concerning subjective interpretations by the designer in the evaluation of the carrying capacity, concludes the work together with possible further developments. 


\section{The standard EN-1065}

\subsection{Classification, strength features}

Five classes of props (A-E) are established. For each type the standard defines the loading capacity $R_{y, k}$ for prop class $y$ at the current extension $l$ :

$$
\begin{aligned}
& R_{\mathrm{A}, k}=51,0 \frac{l_{\max }}{l^{2}} \leq 44,0 k N ; \quad R_{\mathrm{B}, k}=68,0 \frac{l_{\max }}{l^{2}} \leq 51,0 k N \\
& R_{\mathrm{C}, k}=102,0 \frac{l_{\max }}{l^{2}} \leq 59,5 k N ; \quad R_{\mathrm{D}, k}=34,0 k N ; \quad R_{\mathrm{E}, k}=51,0 k N
\end{aligned}
$$

\subsection{Materials and components}

Materials must be in agreement with the existing European standards. All the components must be protected against the corrosion through criteria which affect the designation of a prop: the EN-1065 considers five protection methods, classified according to the manufacturing.

The transversal tube sections must be selected according to the international reference standards. For the props in class $B, C, D$, and $E$ the nominal thickness of any tube (tolerances included) must be no smaller than $2.6 \mathrm{~mm}$; for A class props, it must be $2.3 \mathrm{~mm}$ at least. An overlap between the internal and that external tube of $300 \mathrm{~mm}$ or more is mandatory when the prop is completely extended.

A device is compulsory to avoid that the outside and internal parts of a prop are separated by an involuntary action. A minimal length $-100 \mathrm{~mm}$ between the final part of the outer tube and the internal part of the base when the prop is completely closed - is required to avoid the hand crushing.

\subsection{Design guidelines}

The ultimate strength $R_{y, a c t}$ of a prop by no means can be lower then the loading capacity $R_{y, k}$ at the maximum extension $l_{\max }$. For the props classes $\mathrm{A}, \mathrm{B}$, and $\mathrm{C} R_{y, a c t} \geq R_{y, k}$ must be also tested at any intermediate configuration, including the configuration completely closed; tests must be executed with the inner tube downward as well.

The structural schematization defined by the EN-1065 is depicted in figure 1, in case of covered and uncovered threading. The overlapping zone is modeled by the contemporary presence of the inner and outer tubes, relatively bonded by a hinge (located at point $\mathrm{C}$ by the device of length regulation) and a frictional constraint (at point B of contact among the two tubes). In Authors opinion this last issue is not sufficiently clear: in the present note a perfect constraint has been considered as in figure 1 .

Production defects are modeled as follows. An eccentricity $e_{t}$ at the load application point - taken as independent on the geometry of the prop and of its base - equal to $10 \mathrm{~mm}$; an inclination angle $\Delta \varphi_{0}$ due to the outer/inner tube spacing and to the length of the actual overlapping zone ${ }^{1}$; an initial sinusoidal configuration with a maximal inflection of $\frac{l}{500}$, being $l$ the actual prop length. Props must be designed against buckling of the tubes, taking into account the influence of the actual configuration on the internal actions (according to the second order beam model). The evaluation of the actions in the tubes must follow the principles of elasticity, assuming that the material behavior is linear at all the tension levels. The stress state in the transversal sections of the tubes can be calculated in the framework of perfect plasticity, up to the rise of a plastic hinge.

According to the standard, the slope of the prop base must evolve during the loading process, simulating the progressive failure of a real support; this is one of the three collapse mechanisms indicated by EN-1065. The mechanics of such an evolution is summarized in a torsional elastic constraint, ruled by a non linear constitutive law - see figure 2 - between rotation $\varphi_{b}$ of the base and moment $M_{\text {spring. An eccentricity }}$ $e_{b, 0}=0,40 D_{1}$ models initial defects in the prop base ${ }^{2}$. By the action of external loads the prop base is free to rotate up to $\varphi_{b}=1^{\mathrm{deg}}$. Any further rotation is prevented until the ratio $\frac{M_{\text {spring }}}{N}$ reaches the limit value $e_{b, \text { core }}=-0,25 D_{1}$. For higher values of the ratio, $M_{\text {spring }}$ increases linearly with the base rotation

\footnotetext{
${ }^{1} \Delta \varphi_{0}$ must be evaluated from the nominal device sizes; see an example in section 4.

${ }^{2}$ For plane bases, the base thickness $t$ can be considered as a part of the effective diameter $D_{1}=D+2 t$ having denoted with $D$ the outer diameter of the downward tube
} 


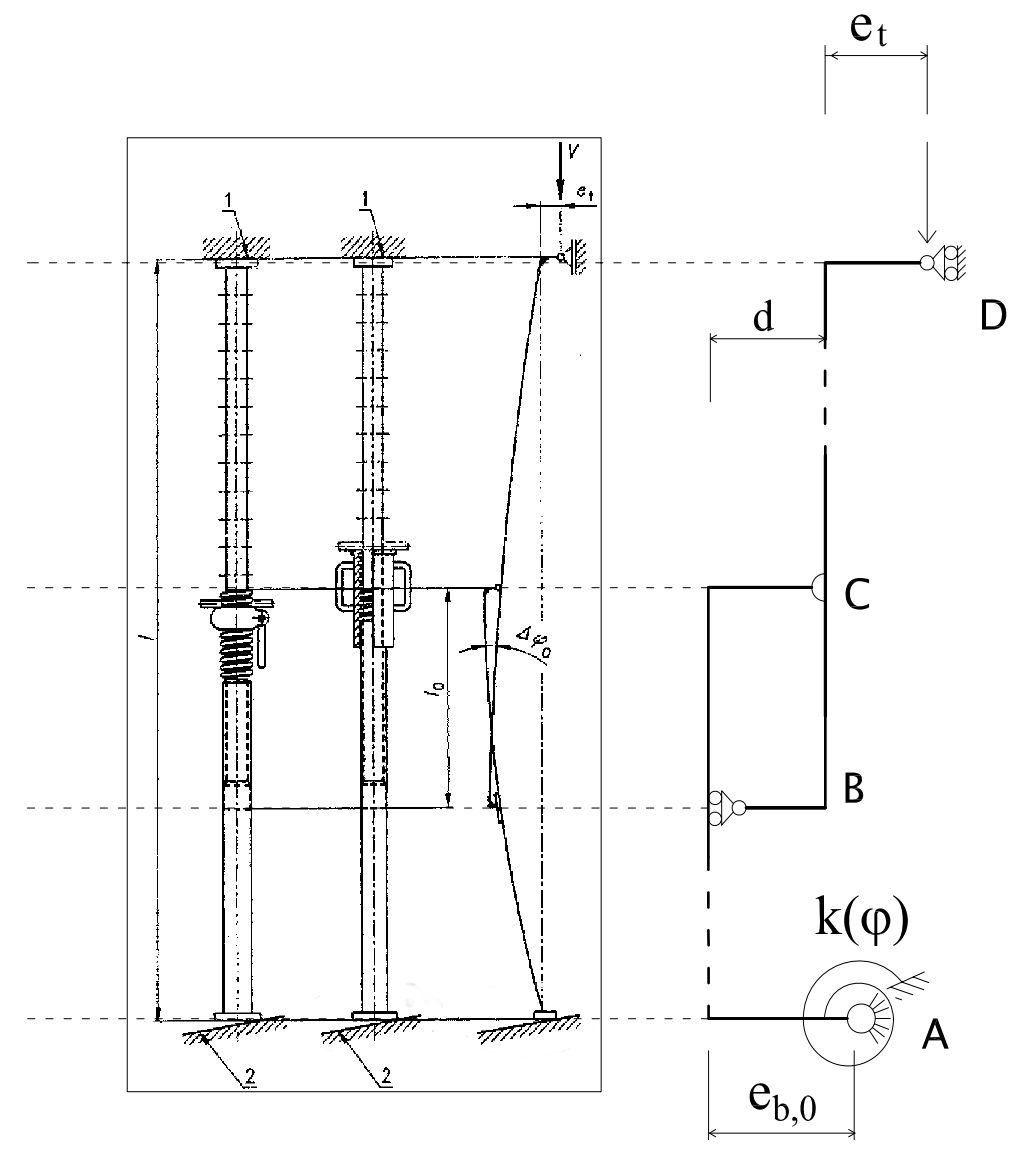

Figure 1: Structural modeling for prop design. In the present note a perfect constraint has been considered at point B instead of a frictional one. Distance $d$ is actually vanishing: it must be conceived as a graphical artefact. Constitutive law $k(\varphi)$ is described in figure 2. Throughout the paper, vertical load $V$ is denoted by the usual letter $P$.
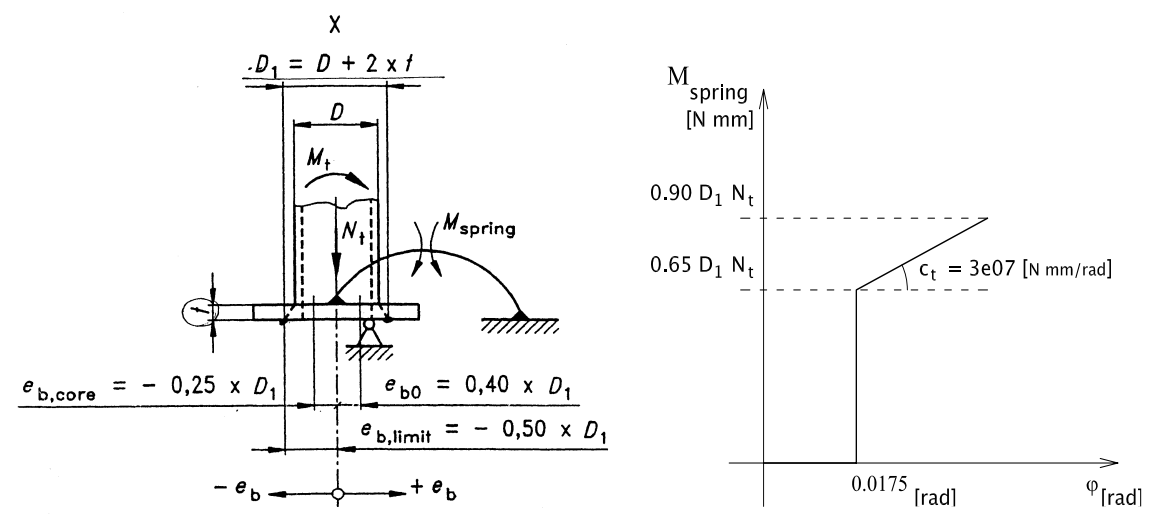

Figure 2: Prop base modeling by means of torsional constraint $M_{\text {spring }}$ versus $\varphi_{b}$.

increment ${ }^{3}$ with stiffness $c_{t}=3 e 07 \frac{\mathrm{Nmm}}{\mathrm{rad}}$ up to a failure value which corresponds to a failure eccentricity $e_{b, \text { limit }}=-0,50 D_{1}$.

\footnotetext{
${ }^{3}$ This is true only for given axial load $N$. See a remark in the conclusion.
} 
Flexural collapse is the third mechanism provided by the EN-1065. The plastic flexural strength $M_{p l, N}$ must be reduced according to the equation:

$$
M_{p l, N}=M_{p l} \cos \left(\frac{\pi}{2} \frac{N}{N_{p l}}\right)
$$

in order to take in to account the axial load $N$ effects. In (3) $M_{p l, N}$ denotes the reduced plastic flexural strength, $M_{p l}$ the plastic flexural strength of the tube section, $N_{p l}$ the compressive strength of the tube section.

\section{An algorithm for ultimate strength assessment.}

A three step algorithm is here described for the prop design. In it, each step corresponds to a branch of the constitutive law for the prop base torsional constraint $k(\varphi)$, as depicted in figure 2 .

In the presence of external actions that induce rotations $\varphi_{b}$ lower than $1^{d e g}$, the prop base torsional constraint has null stiffness and corresponds to a standard hinge (analysis \#1). Once the limit rotation $\varphi_{b}=1^{d e g}$ has been reached, any further rotation is prevented until $\frac{M_{s p r i n g}}{N}=e_{b, c o r e}$. In such a step of the procedure (analysis \#2) the spring stiffness is unbounded and the torsional constraint is equivalent to a full constraint with an imposed rotation $\varphi_{b}=1^{\mathrm{deg}}$. Finally, when external actions induce a ratio $\frac{M_{s p r i n g}}{N} \geq e_{b, c o r e}$ (analysis \#3), the torsional stiffness is constant and equal to $c_{t}=3 e 7 \frac{\mathrm{Nmm}}{\mathrm{rad}}$. This analysis runs until $\frac{M_{s p r i n g}}{N}=e_{b, \text { lim }}$, at which the prop fails due to the exhausted support strength failure mechanism.

At any given prop geometry, three sets of admissible loads are defined, one for each analysis. The prop falls into the first set if $0 \leq P \leq P_{1^{d e g}}$; analysis \#2 is required for $P_{1^{d e g}} \leq P \leq P_{\text {lim }}$ whereas the last analysis runs for $P_{\text {lim }} \leq P \leq P_{\text {fail }}$. Accordingly, the external load is increased from a null initial value until $P=P_{f a i l}$ and, within each interval, internal stresses and strains are deduced from the corresponding structural schematization. Figure 3 summarizes this path of reasoning.
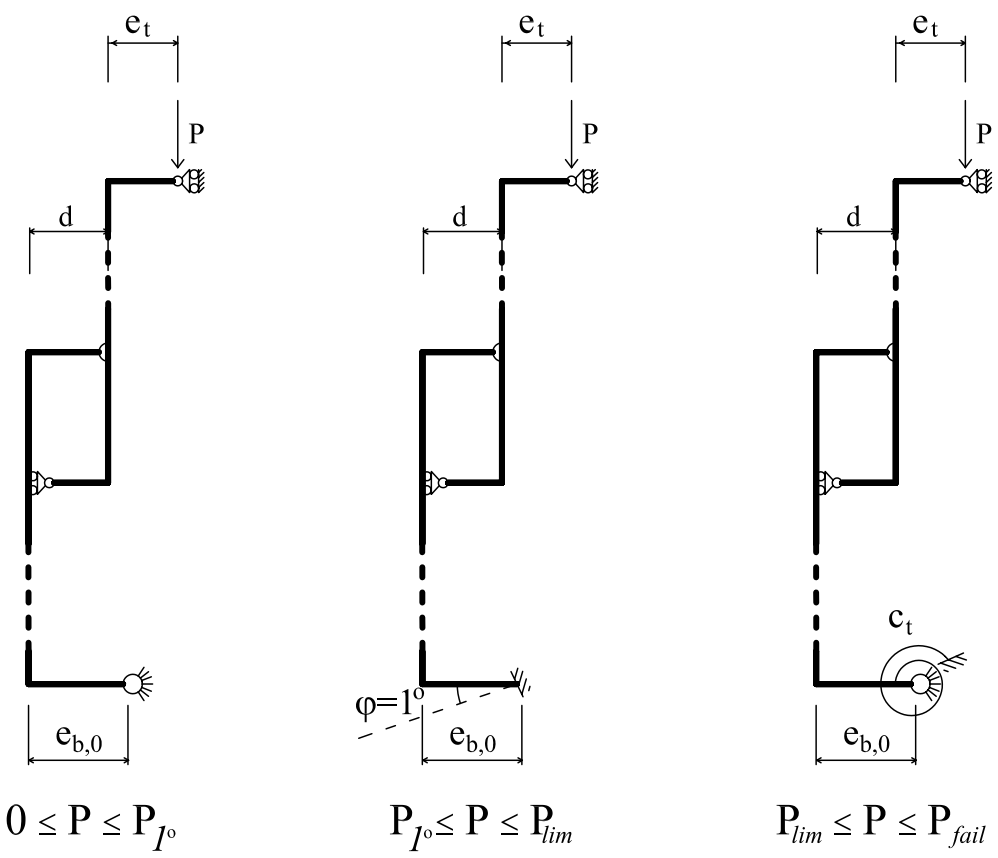

Figure 3: External load intervals for the proposed algorithm. $P_{1^{d e g}}, P_{l i m}$ e $P_{f a i l}$ depend on geometry and on the material properties. 
To evaluate $P_{1 \text { deg }}, P_{\text {lim }}$ e $P_{\text {fail }}$, the structural schematization is subdivided in parts, separating the outer from the inner tube, as in figure 4. The $A B$ interval is the non-overlapping part of the downward tube, whereas $B C$ is its overlapping part. Similarly, $D C$ is the non-overlapping part of the upwards tube, whereas $C B$ is its overlapping part.

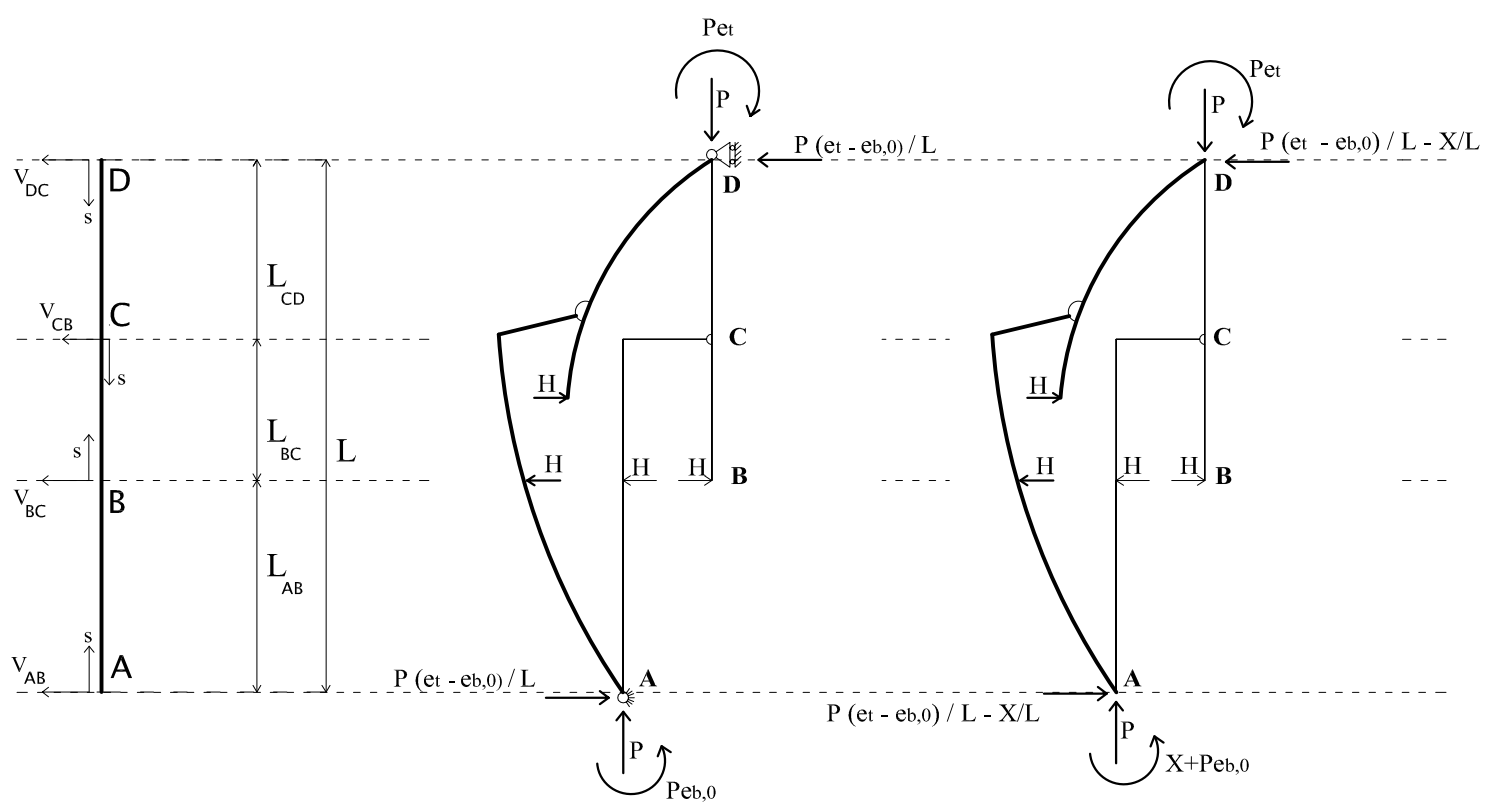
a) notation
b) analysis \#1
c) analysis \#2 and \#3

Figure 4: Notation and elastica configurations.

Functions $v_{n}(s)$ with $n=A B, B C, C D, C B$ describe the prop axis (small) deflection in each part: the Bernoulli-Navier equations of bending read:

$$
\frac{d^{2} v_{n}^{e l}}{d s^{2}}=-\frac{M(s, P)}{E I_{n}} \quad n=A B, B C, C D, C B
$$

- see also figure 4. Boundary conditions ( 8 for analysis \#1 and 9 for analyses \#2 and \#3 which are overconstrained) can be derived from continuity and constraint requirements. For all the three analyses, a linear system of equations comes out; once solved, the elastic contribution ${ }^{4} v_{n}^{e l}$ can be recovered as a function of the external load P.

The values of $P_{1 \text { deg }}, P_{\text {lim }}$ e $P_{\text {fail }}$ can be thereafter straightforwardly evaluated. By imposing a rotation of 1 degree at the bottom hinge in analysis \#1, the value of $P$ corresponding to $P_{1_{\text {deg }}}$ is recovered. By imposing $\frac{M_{\text {spring }}}{N}=e_{b, \text { core }}$ at the bottom full constraint in analysis \#2, one evaluates $P_{\text {lim }}$. Finally, $P_{\text {fail }}$ comes out in analysis \#3 by imposing $\frac{M_{\text {spring }}}{N}=e_{b, \text { limit }}$.

If the loading capacity $R_{y, k}$ - evaluated from equations (1-2) - is lower then $P_{1^{d e g}}$, the displacements and bending moment must be deduced from analysis \#1; if $P_{1^{d e g}} \leq R_{y, k} \leq P_{\text {lim }}$ they turn out from analysis \#2 and if $P_{\text {lim }} \leq R_{y, k} \leq P_{\text {fail }}$ from analysis \#3. If $P_{\text {fail }} \leq R_{y, k}$ then the prop cannot avoid the failure due to the exhausted support strength: geometry and/or materials must be modified properly.

Once the bending moment $M(s, P)$ has been deduced from the structural schematization pertaining to $R_{y, k}$, safety against flexural collapse requires that $M(s, P) \leq M_{p l, N}$ for any $0 \leq s \leq l$, with $M_{p l, N}$ evaluated by (3). Moreover, structural stability requires that $R_{y, k}<P_{E}$ where $P_{E}$ denotes the Eulerian load $^{5}$ of the scheme pertaining to $R_{y, k}$ - see figure 4 .

\footnotetext{
${ }^{4} \mathrm{EN} 1065$ standard defines the total deflection $v_{n}$ as the sum of the elastic contribution $v_{n}^{e l}$, of the initial sinusoidal deflection $v_{n}^{\text {sin }}$ and of the deflection $v_{n}^{\text {inc }}$ due to the inclination angle $\Delta \varphi_{0}$. These last two displacement fields do contribute to the bending moment within the second order theory.

${ }^{5}$ Evaluated at the singularity point of Bernulli-Navier linear system matrix: see equations (10-17) for the example in section 4.
} 


\section{An application}

Assuming $l=l_{\max }$ and the inner tube upwards, the ultimate strength of an A40 steel prop is evaluated. Materials for both tubes is S355JR (Young modulus $E=210 \mathrm{GPa}$, yield strength $f_{y}=355 \mathrm{MPa}$ ). Geometry parameters read - see also figure 5: maximal extension $l_{\text {max }}=l_{o}+l_{m}+l_{i}=4000 \mathrm{~mm}$; overlapping length $l_{m}=300 \mathrm{~mm}$; holes diameter $d_{h}=18 \mathrm{~mm}$; distance between holes $a_{h}=100 \mathrm{~mm}$; diameter at the end of the inner tube $d_{p}=50.5 \mathrm{~mm}$; inner diameter of the thread $d_{s}=54.5 \mathrm{~mm}$; base thickness $t=6 \mathrm{~mm}$.

Outer tube parameters: outer diameter $D_{o}=60.9 \mathrm{~mm}$; effective diameter at the base $D_{o 1}=D_{o}+2 t=$ $72.9 \mathrm{~mm}$; thickness $t_{o}=2.3 \mathrm{~mm}$; length $l_{o}+l_{m}=2100 \mathrm{~mm}$.

Inner tube parameters: outer diameter $D_{i}=52.3 \mathrm{~mm}$; effective diameter at the base $D_{i 1}=D_{i}+2 t=$ $64.3 \mathrm{~mm}$; thickness $t_{i}=2.3 \mathrm{~mm}$; length $l_{i}+l_{m}=2200 \mathrm{~mm}$.
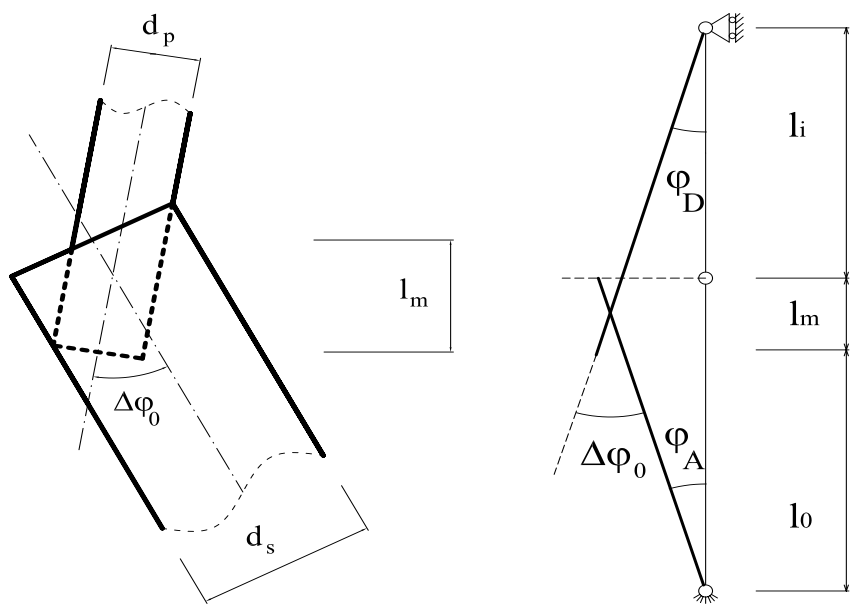

Figure 5: Modeling of the two tubes overlapping. Here: $\Delta \varphi_{0}$ is the angle between the tubes; $l_{m}$ the overlapping length; $d_{s}$ inner diameter at the end of the outer tube; $d_{p}$ outer diameter at the end of the inner tube

Parameters $d_{p}$ e $d_{s}$ (see figure 5) play a basic role in the evaluation of angle $\Delta \varphi_{0}$ between the tubes; making reference to figure 4 , the deflection due to the inclination angle $\Delta \varphi_{0}$ - denoted with $v_{n}^{\text {inc }}(s)$ - reads:

$$
v_{A B}^{i n c}(s)=m_{e} \cdot s \quad v_{B C}^{i n c}(s)=11.9+m_{e} \cdot s \quad v_{D C}^{i n c}(s)=m_{i} \cdot s \quad v_{B C}^{i n c}(s)=12.8+m_{i} \cdot s
$$

where $m_{e}=\tan \varphi_{A} \cong \varphi_{A}=0.00661 \mathrm{rad}$ and $m_{i}=\tan \varphi_{D} \cong \varphi_{D}=0.00672 \mathrm{rad}$ (see figure 5). The standard does not indicate an algorithm to evaluate $\Delta \varphi_{0}$, the following approach has been pursued. The tolerances at the top and at the bottom between the two vertical tubes are defined as:

$$
g_{b o t t}=d_{o}-d_{p} ; \quad g_{t o p}=d_{s}-D_{i}
$$

having set $d_{o}$ the inner diameter of the outer tube and $D_{i}$ the outer diameter of the inner tube. Moving from the vertical position, the tubes relatively rotate about the hinge in figure 1. Geometrical analysis lead to the following first order approximated (i.e. $\tan \varphi=\varphi$ ) equations:

$$
\left\{\begin{array}{l}
\left(l_{o}+l_{m}\right) \cdot \varphi_{A}-\frac{g_{t o p}}{2}=l_{i} \cdot \varphi_{D} \\
l_{o} \cdot \varphi_{A}=\left(l_{i}+l_{m}\right) \cdot \varphi_{D}-\frac{g_{\text {bott }}}{2}
\end{array}\right.
$$

whose solution yields $\varphi_{A}$ and $\varphi_{D}$, whence $\Delta \varphi_{o}=\varphi_{A}+\varphi_{D}$.

The initial sinusoidal deflection $v_{n}^{\sin }(s)$, with notation of figure 4 , reads:

$$
\begin{aligned}
& v_{A B}^{s i n}=\frac{l_{\max }}{500} \sin \frac{\pi}{l_{\max }} s \quad v_{B C}^{\sin }=\frac{l_{\max }}{500} \sin \frac{\pi}{l_{\max }}\left(L_{A B}+s\right) \\
& v_{D C}^{\sin }=\frac{l_{\max }}{500} \sin \frac{\pi}{l_{\max }}\left(l_{\max }-s\right) \quad v_{C B}^{\sin }=\frac{l_{\max }}{500} \sin \frac{\pi}{l_{\max }}\left(l_{\max }-L_{C D}-s\right)
\end{aligned}
$$


The involved tubes geometry has an impact on the inertia moments of the tubes section. For the outer tube, the section is a ring with inertia $I_{\text {out }}=182033 \mathrm{~mm}^{4}$. For the inner one, the standard EN-1065 provides a special procedure for inertia evaluation, summarized in appendix 1: $I_{\text {in }}=87587 \mathrm{~mm}^{4}$ comes out.

According to the Eulerian theory of beams, one gets the reactive forces of figure 4. In analysis \#2 and \#3, $X$ stands for the over-constrain force at the prop base. The reaction force $H$ has to be evaluated in the deformed configuration as a function of the external load $P$, of deflection $v_{D C}\left(L_{C D}\right)$ at point $C$ and eventually of $X$.

In analysis \#1 - $0 \leq P \leq P_{1^{\text {deg }}}$ - the Bernoulli-Navier equations (4) read:

$$
\begin{gathered}
v_{A B}^{\prime \prime}(s)+\alpha_{A B}^{2} v_{A B}(s)=-\alpha_{A B}^{2}\left(m_{e} s+\left(e_{t}-e_{b, 0}\right) \frac{s}{l_{\max }}+e_{b, 0}\right)-\frac{\alpha_{A B}^{2} l_{\max }}{500} \sin \pi \frac{s}{l_{\max }} \\
v_{B C}^{\prime \prime}(s)+\alpha_{A B}^{2} v_{B C}(s)=-\alpha_{A B}^{2} \frac{l_{\max }}{500} \sin \frac{L_{A B}+s}{l_{\max }} \pi+ \\
+\alpha_{A B}^{2}\left[\left(v_{D C}\left(L_{C D}\right)+\frac{l_{\max }}{500} \sin \frac{l_{\max }-L_{C D}}{l_{\max }} \pi+m_{i} L_{C D}+e_{t}-\frac{L_{C D}}{l_{\max }}\left(e_{t}-e_{b, 0}\right)\right) \frac{s}{L_{B C}}+\right. \\
\left.-\left(e_{t}-e_{b, 0}\right) \frac{L_{A B}+s}{l_{\max }}-e_{b, 0}-11,9-m_{e} s\right] \\
v_{D C}^{\prime \prime}(s)+\alpha_{C D}^{2} v_{D C}(s)=\alpha_{C D}^{2}\left(\left(e_{t}-e_{b, 0}\right) \frac{s}{l_{\max }}-m_{i} s-e_{t}\right)-\alpha_{C D}^{2} \frac{l_{\max }}{500} \sin \pi \frac{l_{\max }-s}{l_{\max }} \\
v_{C B}^{\prime \prime}(s)=-\alpha_{C D}^{2}\left(1-\frac{s}{L_{B C}}\right)\left[v_{D C}\left(L_{C D}\right)+\frac{l_{\max }}{500} \sin \pi \frac{l_{\max }-L_{C D}}{l_{\max }}+\right. \\
\left.+m_{i} L_{C D}+e_{t}-\left(e_{t}-e_{b, 0}\right) \frac{L_{C D}}{l_{\max }}\right]
\end{gathered}
$$

with $\alpha_{A B}^{2}=\frac{P}{E I_{\text {out }}}$ e $\alpha_{C D}^{2}=\frac{P}{E I_{\text {in }}}$. Eight boundary conditions are mandatory to solve problem (6-9):

$$
\begin{aligned}
v_{A B}(0) & =0 \\
v_{A B}\left(L_{A B}\right) & =v_{B C}(0) \\
v_{B C}^{\prime}(0) & =v_{A B}^{\prime}\left(L_{A B}\right) \\
v_{B C}(0) & =v_{C B}\left(L_{B C}\right) \\
v_{D C}(0) & =0 \\
v_{D C}\left(L_{C D}\right) & =v_{C B}(0) \\
v_{C B}^{\prime}(0) & =v_{D C}^{\prime}\left(L_{C D}\right) \\
v_{C B}(0) & =v_{B C}\left(L_{B C}\right)
\end{aligned}
$$

The limit load $P_{1^{\text {deg }}}$ for analysis \#1 satisfies the condition $v_{A B}^{\prime}(0)=\frac{\pi}{180}$ : it amounts to $P_{1^{\text {deg }}}=5,49 \mathrm{kN}$. By imposing a null matrix determinant in the system of equations (10-17) the critical load for analysis \#1 turns out to be $P_{c r, 1}=15,32 \mathrm{kN}$.

Algorithms for analysis \#2 and \#3 follow the same path of reasoning. They require a further boundary condition because of the over-constraint. In the second scheme the rotation at the base must be one degree.

$$
v_{A B}^{\prime}(0)=\frac{\pi}{180} ;
$$

In the third scheme, the moment at the base is related to the rotation in the spring by means of elasticity constant $c_{t}$ - see figure 2:

$$
v_{A B}^{\prime}(0)=-\frac{X}{c_{t}}-\frac{\left(e_{b, 0}+e_{b, c o r e}\right) P}{c_{t}}+\frac{\pi}{180}
$$

The transition between analysis \#2 and \#3 is stated by the condition

$$
v_{A B}^{\prime}(0)=\frac{\pi}{180} \rightarrow X=-P\left(e_{b, 0}+e_{b, c o r e}\right)
$$


whence $P_{\text {lim }}=10,25 k N$. The support strength of the prop is exhausted when $X=-P\left(e_{b, \lim }+e_{b, 0}\right)$, and $P_{\text {fail }}$ turns out to be $13,64 k N$. By imposing a null matrix determinant in the system of nine boundary conditions, the critical loads for analyses \#2 and \#3 come out. They amount to $P_{c r, 2}=29,44 k N$ and $P_{c r, 3}=21,45 k N$.

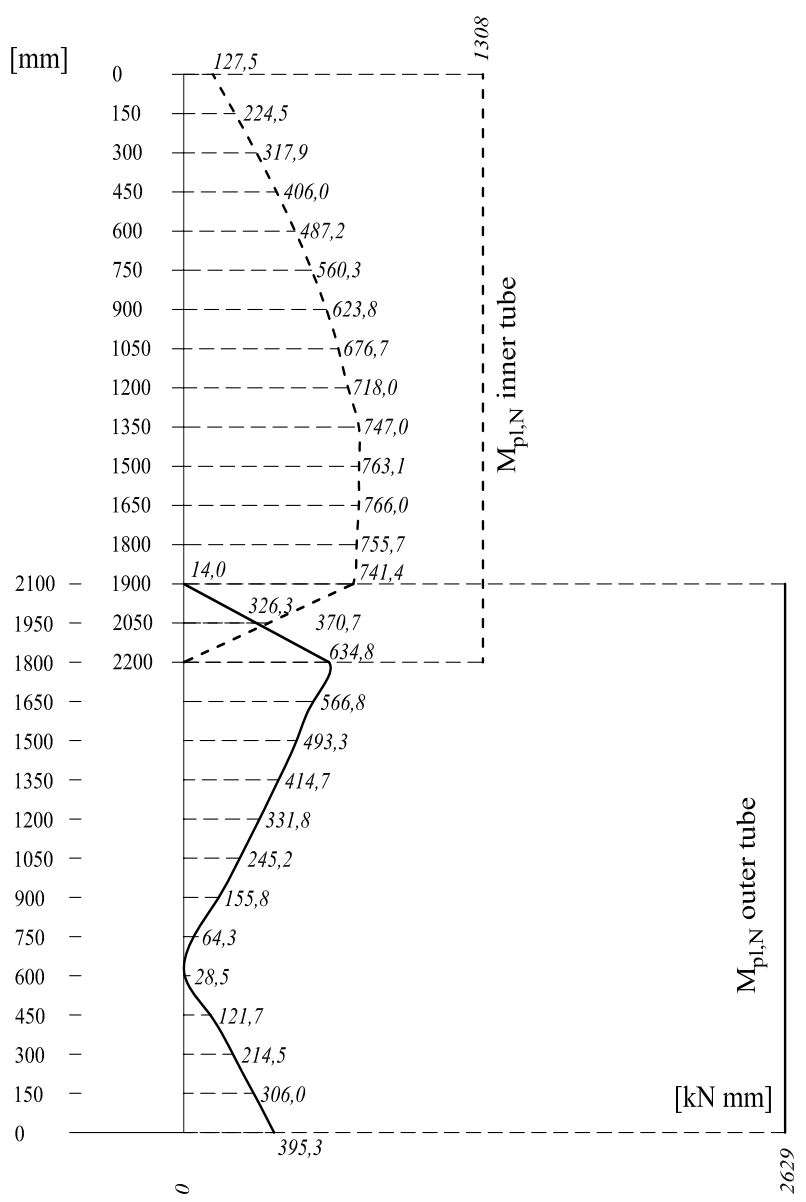

Figure 6: Comparison between the plastic flexural strength $M_{p l, N}$ and the (absolute value of) actual bending moment.

The ultimate strength $R_{\mathrm{A}, a c t}$ of a prop by no means can be lower then the loading capacity $R_{\mathrm{A}, k}$ evaluated from equation (1):

$$
R_{\mathrm{A}, k}=51.0 \frac{l_{\max }}{l^{2}}=51.0 \frac{4}{16}=12.75 \mathrm{kN}
$$

In view of the inequality:

$$
R_{\mathrm{A}, k}=12.75 k N<15.32 k N=P_{c r, 1}=\min _{n=1,2,3} P_{c r, n}
$$

it can be concluded that the ultimate strength is adequate against the failure due to structural instability. It is also adequate against failure at the base of the prop, for being

$$
R_{\mathrm{A}, k}=12.75 k N<13.64 k N=P_{\text {fail }}
$$

Finally, because $P_{\text {lim }}<R_{\mathrm{A}, k}<P_{\text {fail }}$, it is mandatory to check that the bending moment due to $P=R_{\mathrm{A}, k}$ in analysis \#3 is lower than $M_{p l, N}$ at all points of the prop. The plastic flexural strength, according to equation (3), amounts to $M_{p l, N}=2629226 \mathrm{Nmm}$ for the outer tube and to $M_{p l, N}=1280789 \mathrm{Nmm}$ for the inner one. A plot of bending moment versus the plastic flexural strength is represented in figure 6 . Its analysis allows to conclude that, at the maximum extension, the analyzed prop is in agreement to standard 
EN-1065. The analysis of the carrying capacity according to EN-1065 is mandatory at the minimum length and at the most unfavorable intermediate configuration as well. Analyses must to be repeated positioning the prop with the internal tube downward.

It seems interesting to plot (figure 7) the elastic contribution to the deflection: the effect of the constraints imposed in the three phases of the load process is clear. During analysis \#1 the rotation at point $A$ is free, while it is prevented in analysis \#2: in the latter, the slope of the deflection curve at point $A$ in figure 7 is constant and equal to $1^{\mathrm{deg}}$. The deflection at the end of analysis \#2 (corresponding to an external load of about $75 \%$ of $\left.P_{\text {fail }}\right)$ is about $58 \%$ of the ultimate deflection. As expected therefore, most of the deflection takes place in analysis \#3.

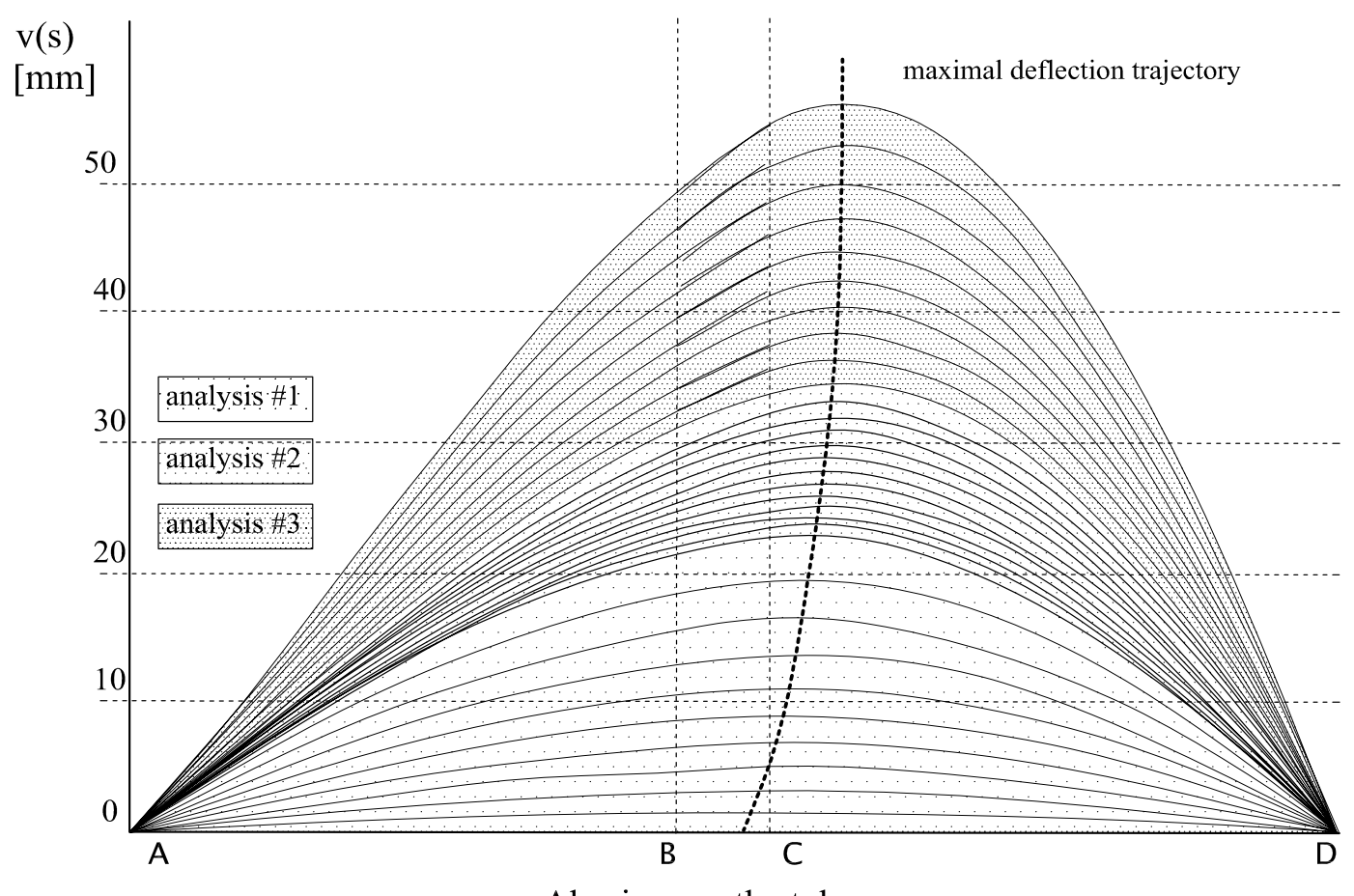

Abscissa on the tubes

Figure 7: Elastic deflection $v^{e l}(s)[\mathrm{mm}]$ as a function of the external load up to the carrying capacity $P_{\text {fail }}$. One notes - at point $A$ - the effect of changing constraints in the three analyses. The non rectilinear trajectory of the point of maximum deflection is noticed, whose knowledge seems of interest, for instance, in order to the deflection measure in the evaluation of the carrying capacity through experimental tests.

\section{Conclusions}

Target of this note was the design of adjustable telescopic steel props, evaluating their carrying capacity in agreement to the standard EN 1065. The carrying capacity is defined by the standard as to the minimum value of the external load which is able to activate one of the following three mechanisms: the collapse due to Eulerian instability [1], to exhausted support strength, and to flexural failure of the tubes. For the evaluation of the carrying capacity a strategy was suggested; it stems from the non linear modeling of the torsional spring that aims at reproducing the prop base behavior. The algorithm analytically describes the evolution of the structural response while the external load increases from the initial null value up to the loading capacity $R_{y, k}$, required to be lower than the ultimate strength $R_{y, \text { act }}$.

The methodology described in this work has been implemented in a computer code, named PrOptimizer ${ }^{6}$. The code was successfully used for the optimization of the steel prop production of an Italian

\footnotetext{
${ }^{6}$ A companion code, named PrCertifier, has been used for the certification according to the Germany DIN-EN-1065 standard. Both
} 
company: in the set of the props in agreement with EN-1065, minimum weight props were determined. The optimum products were subject to further constraints (commercial dimension of tubes, further company requirements): the outcomes of beyond 200.000 possible props are not publishable. In section 4 an application was shown with data concerning the real production.

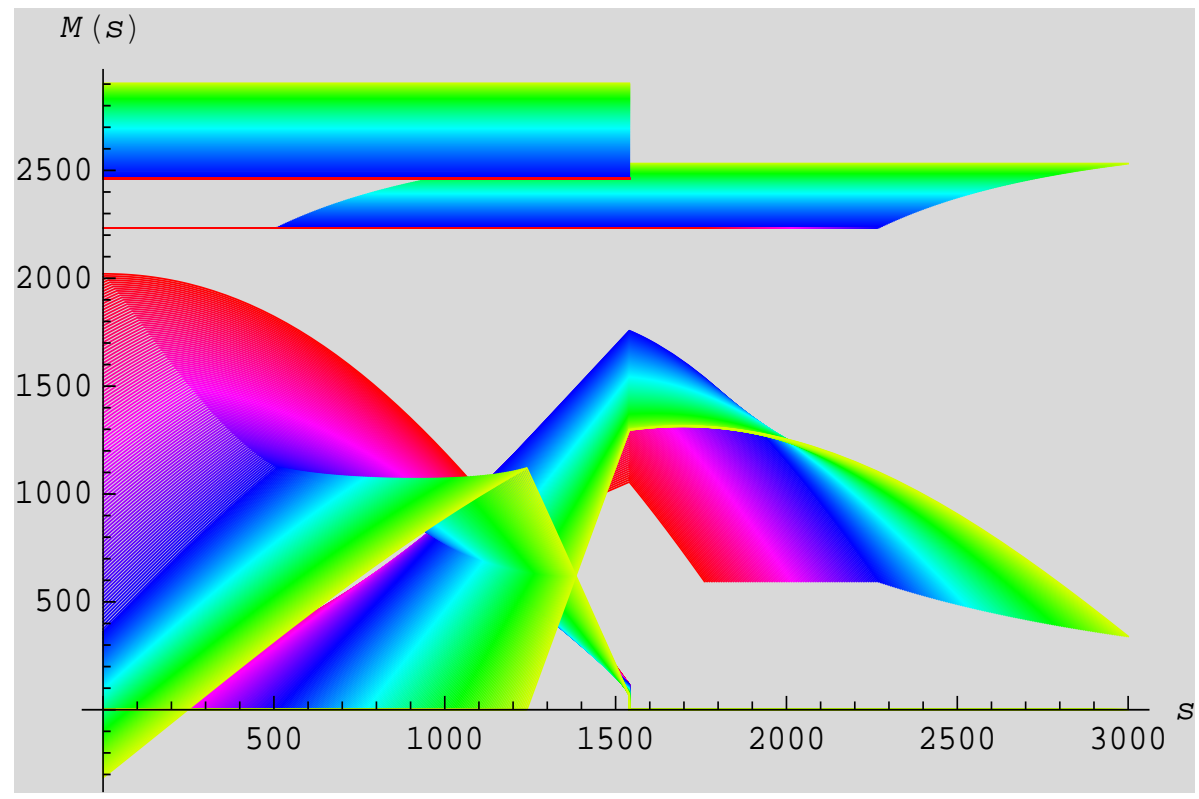

Figure 8: A comprehensive plot of bending moment $[\mathrm{kN} \mathrm{mm}]$ in the tubes at different tube lengths [mm] and a comparison with $M_{p l, N}$ (the upper plateau) defined by formula (3). The plot, one of the outcomes of code PrOptimizer, refers to a C30 test with outer tube downward. Red curves refer to the minimum extension, whereas yellow curves to the maximum one.

In conclusion, it might be useful to highlight a few issues in the standard which require subjective interpretations by the designer in the evaluation of the carrying capacity. The structural schematization of figure 1 is not of immediate comprehension in the tubes overlapping zone neither from the graphic point of view, in particular the crossing of the tubes, nor from the mechanics - the frictional constraint description is largely inadequate. The standard does not give any direction for the evaluation of the tilt angle $\Delta \varphi_{0}$; the eventual relationship between the angle at the base $\varphi_{A}$, the sinusoidal initial deflection, and the modeling of the prop support is not specified. In the latter, there is a lack in the norm of the motivations for the adopted constitutive law and its independency on the base and of the tubes configuration, at least with reference to the spring constant $c_{t}=3 e 7 \mathrm{Nmm} / \mathrm{rad}$. It seems to of interest noting that such a constitutive law, namely:

$$
\varphi_{b}=-\frac{M_{\text {spring }}}{c_{t}}-\frac{\left(e_{b, 0}+e_{b, \text { core }}\right) N}{c_{t}}+\frac{\pi}{180}
$$

is linear for a given external load $P$. Whereas the load increment history is taken into account, the real relationship between moment $M_{\text {spring }}$ and rotation $\varphi_{b}$ is no longer proportional - see figure 9 .

Further developments are in progress. The assumption of small strains seems to be inappropriate at least in analysis \#3 $P_{\text {lim }} \leq P \leq P_{\text {fail }}$ for some flexible props. Perhaps, the large deformation beam theory [3], might explain more precisely the influence of the external load on the bending moment. This issue is motivated also from experimental investigations: for some classes of props, design from point 9.2 of EN-1065 appear to be more conservative than prop certification via experimental tests.

codes are property of the Authors at the University of Brescia. 


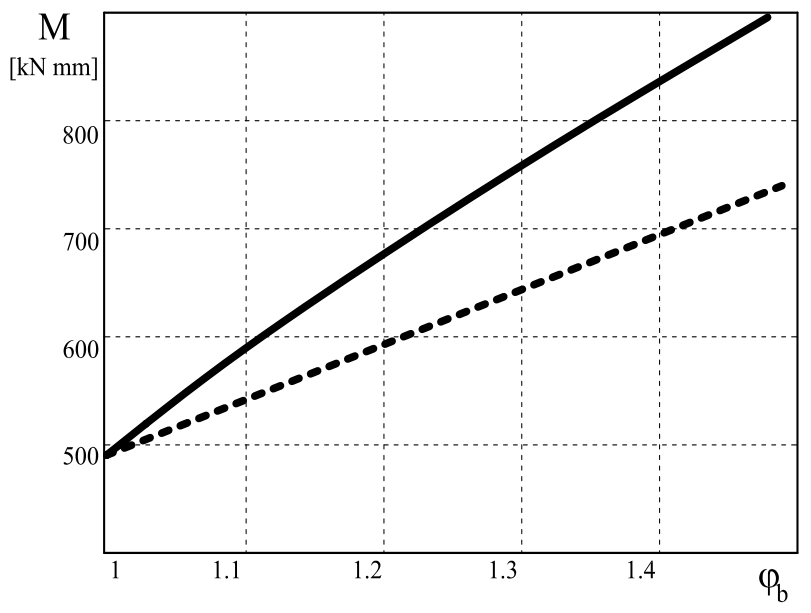

Figure 9: Relationship between $M_{\text {spring }}$ and rotation $\varphi_{b}$ at $P_{\text {lim }} \leq P \leq P_{\text {fail }}$ (analysis \#3): the dashed line comes from the standard, the solid line is the "actual" one.

\section{Acknowledgments}

The present work and the two codes PrOptimizer, PrCertifier would not have been successful without the cooperation of several students, which we heartily acknowledge: S. Modena, F. Colosio, R. Marzi, A. Pansini, A. Mita. It has been motivated and supported by the company Ferro-met S.r.l.: we are grateful indebted with Dr. C. Boninsegna for his strong and warm cooperation.

\section{References}

[1] Q. Nguyen, Stability and nonlinear solid mechanics, John Wiley and Sons B.V. (2000).

[2] N. Panzeri, C. Poggi, Analisi numeriche e sperimentali per la determinazione della capacità portante di puntelli metallici in accordo alla norma uni-en 1065., Costruzioni metalliche, 54, (2002), 1-11.

[3] S. Antman, Nonlinear Problems of Elasticity, Springer (2004).

\section{Appendice 1 - Inertia moments}

Numbers in the present appendix refers to the example in section 4. The external tube inertia moment is evaluated by:

$$
I_{\text {out }}=\frac{\pi}{64}\left(D_{o}^{4}-d_{o}^{4}\right)=182033 \mathrm{~mm}^{4}
$$

The presence of the holes in the inner tube makes the definition and the evaluation of the inertia quite involved. The standard suggests the following equation for $I_{n}$ - see also figure 10 - in the presence of the holes:

$$
I_{n}=\frac{6 \varphi_{\text {out }} R-d\left(3+2 \sin ^{2} \varphi_{\text {out }}\right) \sin \varphi_{\text {out }}}{12} R^{3}-\frac{6 \varphi_{\text {in }} r-d\left(3+2 \sin ^{2} \varphi_{\text {in }}\right) \sin \varphi_{\text {in }}}{12} r^{3}=62494 \mathrm{~mm}^{4}
$$

where:

$$
\varphi_{R}=\arccos \frac{d}{2 R}=1^{r a d} .219 ; \quad \varphi_{r}=\arccos \frac{d}{2 R}=1^{r a d} .184
$$




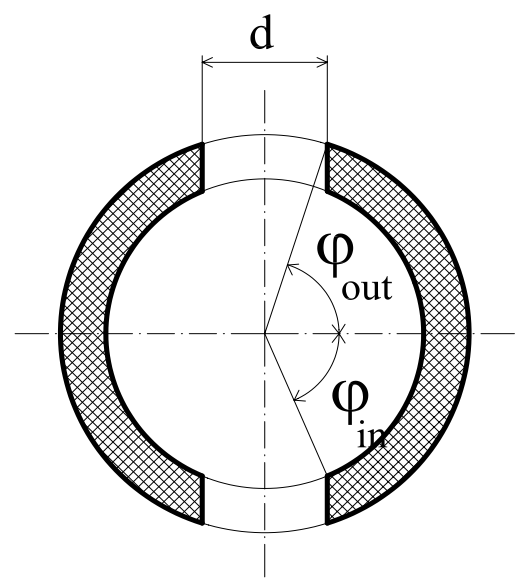

Figure 10: A section of the inner tube.

and $R=\frac{D_{i}}{2}, r=\frac{D_{i}}{2}-t_{i}$ are the outer and inner radius of the tube, respectively. Because holes do not cover the inner tube completely, the inertia moment $I_{i n}$ of a generic section is established by the standard as:

$$
I_{i n}=I_{g r} \frac{1}{1+2 \frac{d}{a_{h}}\left(\frac{I_{g r}}{I_{n}}-1\right)}=87587 \mathrm{~mm}^{4}
$$

where $I_{g r}$ denotes the inertia of a section with no holes:

$$
I_{g r}=\frac{\pi}{4}\left(R^{4}-r^{4}\right)=113140 m^{4}
$$

and $a_{h}$ is the distance between two consecutive holes. 